\title{
Children's altruism in public good and dictator experiments
}

William T Harbaugh; Kate Krause

Economic Inquiry; Jan 2000; 38, 1; ABI/INFORM Global

pg. 95

\section{CHILDREN'S ALTRUISM IN PUBLIC GOOD AND DICTATOR EXPERIMENTS}

\author{
WILLIAM T. HARBAUGH and KATE KRAUSE*
}

\begin{abstract}
We examine the development of altmistic and free-riding behavior in 6-12-year-old children. We find that the level of altruistic behavior in children is similar to that of adults but that repetition has a different effect. Younger children's contributions tend to increase in later rounds of the experiments, whereas the contributions of older children, like those of adults, tend to decline. Group attachment is associated with higher contributions. Contributions in a subsequent dictator experiment are correlated with first-round contributions in the public good experiment, but are not strongly correlated with last-round contributions. (JEL H41)
\end{abstract}

\section{INTRODUCTION}

Research on altruism among adults using linear public good experiments has established a number of interesting results. Adults are initially far more generous than would be true if they were motivated by plain selfishness. With repetition, most gradually start to free-ride, but many continue to contribute substantial amounts, suggesting that a taste for altruism is, if not universal, at least widespread. The existence of such a preference is confirmed by a wide variety of behaviors in nonexperimental settings. Since altruistic behavior is an important feature of the economy, a natural, and important, question is to ask were it comes from. As a first step toward addressing this question, in this article we examine the behavior of 6-to 12-yearold children in public good experiments.

We begin by comparing the extent of altruistic behavior in children with that of adults. If even young children behave in a

* This research was funded by grants from the University of New Mexico Research Allocation Committee and the National Science Foundation. We thank Jim Andreoni, Arik Levinson, Lise Vesterlund, Jim Ziliak. and an anonymous referee for helpful comments. Wo would also like to express our appreciation to the City of Albuquerque recreation program leaders for their help organizing and conducting these experiments, and to give our thanks to the children who participated.

Harbaugh: Assistant Professor, Department of Economics, University of Oregon, Eugene, Oregon 97403-1285. Phone 541-346-1244. Fax 541-346-1243, E-Mail harbaugh a oregon.uoregon.edu

Krause: Assistant Professor, Department of Economics, 1915 Roma NE, University of New Mexico, Albuquerque. New Mexico 87131-1101, Phone 505-2773429. Fax 505-277-9445, E-Mail kkrause(a unm.edu way similar to adults, the taste for altruism must be, if not innate, at least determined by very early experiences. Next, since heterogeneity in altruistic behavior may be due to differences in these experiences, we test whether the variance in children's altruistic behavior is correlated with demographic and other variables. The observed decline in contribution among adults suggests that at least some adults learn the free-riding strategy over the course of the experiment or that confusion regarding the protocol is reduced with repeated play. There is evidence that adults are confused by the experimental protocol, and it is plausible that children might be even more so. To determine whether learning about either the free-riding strategy or about the protocol is age-related, we investigate how behavior in this experiment differs across children of different ages. Finally, to test whether the linear public goods game is a reasonable way of studying altruism in children, we conduct a second test of altruism, based on the dictator game, on a subset of the original subjects.

In a typical linear public goods experiment subjects are recruited and put into groups of $N$ members, where $N$ is generally between 4 and 10. Subjects play either a preannounced or randomly determined number of rounds. At the beginning of each round, subjects are endowed with experiment currency that is exchanged for cash at the end of the experiment. They then must decide whether to keep it or to contribute any portion of it to the group. The money 
given to the group is multiplied by a number $\alpha$ greater than 1 but less than $N$, and then the total is divided equally among the group members. In this game, the individually rational strategy (for selfish preferences) is to donate nothing in each round, while the Pareto optimal result is for each person to donate everything.

Ledyard [1995] provides more information on these experiments and summarizes the conclusions. The result is typically that people donate between one-third and two-thirds of their endowments to the group, that they donate more if $\alpha / N$ (the marginal private return) is larger, and that donations decrease with repetition, though not to zero. These results are generally taken as proof that the subjects have a taste for altruism. The overwhelming majority of these experiments have been conducted using college undergraduates, although one of the earliest studies, Marwell and Ames [1981] used high school students.

We have been able to find only one existing study by economists that is concerned with the altruistic behavior of children. This article, by Peters, Unur, Clark, and Schulze [1997], uses linear public good experiments to test children's altruism toward family members, focusing on the rotten-kid theorem. They find that in this setting children give less than parents and that children's contributions generally do decline with repetition. Developmental psychologists have done a considerable amount of work on the altruistic behavior of children. Eisenberg and Mussen [1989] has a good review of this. Underwood and Moore [1982] report that age has generally been found to be positively correlated with both frequency and levels of contributions. They also note that gender has been found to be correlated with generosity more often than not. Measures of "prosocial" behaviors and advanced levels of "moral judgment" on the part of children have also been associated with generosity. The methodology used in studies by developmental psychologists varies widely and is often very different from that typically used in economic experiments on adults. For example, Grusec [1982] uses mother's reports about children's home behavior. The experiments often ask subjects to make hypothetical choices rather than choices with real consequences and seldom incorporate repe- tition. In contrast, the experiments we report in this article were conducted in a controlled environment that provided for anonymity and real consequences, making them very similar to those that economists have conducted on adults.

One problem with using the linear public good experiment to estimate altruism is that. for selfish players, the Nash equilibrium is to give nothing, and negative donations are impossible. This means that any mistakes will be counted as altruism. While the original motivation for repeating the game was so that subjects' mistakes would be reduced by learning, two recent articles have addressed the issue of confusion more directly. By paying subjects according to their relative earnings, or rank, as opposed to their absolute earnings, Andreoni [1995] alters the standard public goods experiment so that confusion is the only motive for contributing. Palfrey and Prisbey [1998] include parameters for which the Nash equilibrium contribution is positive. Both find a substantial amount of confusion, even after 10 repetitions, but also a substantial amount of altruism.

If children are more likely than adults to make mistakes, or to be slower at learning, then the public goods experiment will tend to overestimate children's altruism relative to that of adults. Although we felt that the protocols used by Andreoni and by Palfrey and Prisbey to compute measures of the effect of confusion would be very difficult to implement with children, we did run a simple test of the consistency of the behavior of our subjects across two different protocols. At three sites, we followed the public good experiment by having children play a simple dictator game, modeled after Andreoni and Miller [1998], that asked them to make donate/keep decisions under varying pricing conditions. We argue that, because the incentives are very clear, errors in the dictator game are likely to be small and that furthermore there is no reason they should be biased in either direction. So, behavior in these two games should be highly correlated, unless it is diminished by confusion in the public good game.

We begin by discussing the design of the public good experiments in Section II. Section III gives the data and results. Section IV discusses the dictator game, and Section V concludes. 


\section{PUBLIC GOODS EXPERIMENT DESIGN}

In each of the experiments reported below, children were randomly assigned to groups of six. Two treatments were used. For the first treatment, $\alpha$ was set at 2, making for a marginal private return to the donor (MPR) of one-third of a token for every token contributed. For the second treatment, $\alpha$ was set at 4 , making for a MPR of twothirds. We call these the low-MPR and highMPR treatments, respectively. Participants were first through seventh-graders recruited at after-school and summer recreational programs in Albuquerque, New Mexico.

Instead of cash, we gave our subjects an endowment of five white poker chips before each round. They were told that at the end of the experiment they would be able to use these tokens to purchase goods such as fancy pencils, small stuffed animals, super balls, and toy airplanes from a store that we set up at the site. The exchange value of one token was about 10 cents. In what can only be described as a very successful effort to increase the salience of the rewards, subjects were shown the goods available at the store in advance. Using tokens and a store seems particularly suitable with younger children, who may have trouble converting cash into goods. (A large majority of parents reported that there was no neighborhood store at which their children were able to shop unsupervised.) Since, according to the surveys, our subjects averaged about $\$ 2$ in weekly allowance, the payoffs involved were quite large in relative terms. Our subjects typically doubled or tripled their disposable incomes for the week.

The subjects were seated behind partitions and were assured that all their actions would be confidential and that we would never disclose who was in what group. They were given a cup to keep their earnings in and a padded manila envelope marked with their identification number to be used for contributions. At the beginning of each round, their five-chip endowments were placed in front of them, and they then placed the chips they wanted to keep in their cup and the chips they wanted to contribute in the envelope. After each round, the envelopes were collected, the contents were poured into a bowl along with the contributions from the other members of their group, and the pooled contents were then doubled (or quadrupled, for the high-MPR treatment). The contents of the bowl were then evenly divided among the group members and returned in the envelopes. (Fractional payoffs were made in red chips worth onethird of a white chip.) They were then asked to count out the returned tokens and place them in their cup. When this was done, we distributed five new tokens to each participant and started the next round.

We emphasized that each token they contributed to the group would result in every person in the group getting one-third (twothirds in the high-return treatment) of a token and that, therefore, contributing a token would mean less for them personally but more for the group. We also acted out two different scenarios, showing that, when everyone donated, the group got more but that any one member could do even better by not donating. While our subjects probably have less cognitive ability than the typical undergraduate subjects, we believe that these instructions were substantially clearer, particularly about the rewards to free-riding, than usual.

We conducted two basic sets of these experiments. The first set was conducted at five schools, with between 17 and 23 children participating at each. In these experiments. the children were not told how many repetitions would be conducted, and the actual number conducted varied from four to eight. When the number of children was not divisible by six, we constructed a synthetic group for the extra subjects, using the decisions of members of another group to determine total contributions for the synthetic group.

The second set of experiments was conducted at eight different schools, with between 12 and 23 subjects participating at each school. In these experiments, when the number of subjects was not divisible by six, the extra subjects were allowed to participate, but the data were not used. Subjects were told in advance that there would be 10 repetitions and were reminded again just before the last iteration. ${ }^{\text {. }}$

1. We exclude data from two additional sites because the number of subjects present, five and seven. was too small to ensure any degree of anonymity regarding group mombership. 
III. LINEAR PUBLIC GOOD EXPERIMENTS: DATA AND RESULTS

We use these data to address four basic questions. First, we compare overall contributions by children with contributions by adults, as reported by other researchers. Next we ask whether observable characteristics of the subjects are associated with altruistic behavior. To do this, we concentrate on contributions in the first round of each experiment. These may be the clearest measures of altruistic taste, since contributions are not influenced by results of previous rounds. ${ }^{2}$ Where possible, we use data from both sets of experiments in this part of the article. ${ }^{3}$ The results are reported below.

Then we look at behavior across iterations in order to ask whether children learn to free-ride. For this analysis, we use only the data from the second set of experiments, because these all had 10 iterations and a preannounced ending, as is typical with the experiments others have done on adults. We also excluded the data from the synthetic groups, because the subjects in these groups were not interacting with each other. These results are reported below.

Last, in Section IV, we compare behavior in the revealed preference public good experiment with that in the voluntary contributions public good experiment. This comparison is based on data from the three sites where we collected both types of data. Our objective here is to address the consistency

2. However, this is also the round that is most likely to be susceptible to subject confusion, and so below we examine similar questions for all rounds.

3. Since we collected somewhat different sets of survey data for the different sets of experiments, some regressions use only data from one set of cxperiments. of behavior across experiments. Inconsistency would suggest that our subjects are confused by one or the other protocol or that the protocols test different things.

\section{First-Round Contributions}

First-round contributions are the simplest to analyze, since there is no previous behavior to consider. ${ }^{4}$ Table I shows the frequency distribution for first round donations. Only about $16 \%$ of the subjects contribute zero, while about $12 \%$ contribute the maximum possible five tokens. Table II shows basic summary data for each of the 12 sites from which we use the data, ordered by MPR. The distribution of contributions are comparable to those from experiments on adults. Mean first-round contributions for subjects at sites with the higher marginal private return were 0.47 tokens, or $24 \%$ higher than those for those at sites with the low MPR. This difference, which Ledyard [1995] calls one of the "strong effects" to be found in public goods experiments, is significant at the 0.05 probability level using a $t$-test.

Although we are encouraged at finding this effect with children, we cannot reject the hypothesis that it is the result of uncontrolled differences between the subjects. Obviously there is a substantial amount of variation in contributions that is unrelated to the MPR. We use regressions in order to control

4. We combine the data from both sets of experiments for most of this analysis. To account for the possibility that the lack of a defined end point affected first-round behavior, we ran the regressions with an indicator variable for the experiment set. This variable was insignificant, and the coefficients on the other variables changed very little.

TABLE I

Frequency Distribution for First-Round Contributions ( $n=208)$

\begin{tabular}{ccc}
\hline Contribution $\boldsymbol{\mu}$ & Frequency & Percentage \\
\hline 0 & 34 & 16 \\
1 & 44 & 21 \\
2 & 62 & 30 \\
3 & 31 & 15 \\
4 & 12 & 6 \\
5 & 25 & 12 \\
\hline
\end{tabular}


TABLE II

Descriptive Data and First-Round Contributions by Site

\begin{tabular}{lccccc}
\hline & & & & \multicolumn{2}{c}{ First-Round Contributions } \\
\cline { 5 - 6 } Site & MPR & Subjects & Rounds & Mean & Standard Deviation \\
\hline 1 & $1 / 3$ & 24 & 6 & 2.2 & 0.88 \\
3 & $1 / 3$ & 17 & 4 & 2.2 & 1.4 \\
5 & $1 / 3$ & 24 & 8 & 1.7 & 1.7 \\
7 & $1 / 3$ & 18 & 10 & 1.8 & 1.5 \\
8 & $1 / 3$ & 18 & 10 & 2.4 & 1.6 \\
10 & $1 / 3$ & 12 & 10 & 0.75 & 0.87 \\
13 & $1 / 3$ & 12 & 10 & 1.8 & 1.5 \\
14 & $1 / 3$ & 12 & 10 & 2.1 & 1.9 \\
Low-MPR average & & & & 1.9 & 1.5 \\
2 & $2 / 3$ & 17 & 6 & 2.4 & 0.79 \\
6 & $2 / 3$ & 18 & 10 & 2.4 & 1.4 \\
9 & $2 / 3$ & 18 & 10 & 3.0 & 1.8 \\
12 & $2 / 3$ & 18 & 10 & 1.8 & 1.8 \\
High-MPR average & & & & 2.4 & 1.5 \\
Overall average & & & & 2.1 & \\
\hline
\end{tabular}

for some possible sources of this variation, and to examine what sorts of factors affect donations. This use of regressions is relatively rare in economic experiments, but it is useful here because there is more variation in the characteristics of our subjects than is typical and because one objective of this article is to see whether this variation is correlated with behavior. Table III shows summary statistics for the variables we will use in the regressions, including some variables we will use later in the paper. Table IV presents ordinary least squares results, with the dependent variable being the log of the contribution plus one. ${ }^{5}$ Those independent variables that are transformed are indicated in Table IV. We also estimated the models using untransformed data, getting the same signs on the statistically significant coeffi-

5. Because contributions are censored at zero and five, two-sided tobit would be a more appropriate statistical procedure, both in these section and the subsequent section which uses a panel of data to examine behavior over iterations. Since to our knowledge there is no statistical routine available for calculating two-tailed tobit for the panel data model, we report the OLS results here instead, for comparability. We ran these regressions using the tobit model, with very similar results.

TABLE III

Summary Statistics for Regression Variables

\begin{tabular}{lccccc}
\hline Variable & Observations & Mean & Standard Deviation & Min & Max \\
\hline Tokens shared, first round & 208 & 2.1 & 1.5 & 0 & 5 \\
High MPR site & 208 & 0.34 & 0.48 & 0 & 1 \\
Attendance & 208 & 0.59 & 0.42 & 0 & 1 \\
Age in years & 201 & 8.8 & 1.6 & 5.6 & 14 \\
Male & 206 & 0.47 & 0.50 & 0 & 1 \\
Number of siblings & 189 & 1.4 & 0.92 & 0 & 5 \\
Single parent family & 111 & 0.25 & 0.44 & 0 & 1 \\
Allowance in dollars per week & 166 & 2.1 & 2.7 & 0 & 10 \\
TV watching in hours per week & 58 & 11 & 6.0 & 0 & 35 \\
Church attendance (times in an & 58 & 1.1 & 1.8 & 0 & 8 \\
average month) & & & & \\
\hline
\end{tabular}


TABLE IV

First Iteration Contributions: OLS Regression Results

\begin{tabular}{lccc}
\hline & & \multicolumn{2}{c}{ Regression } \\
\cline { 2 - 4 } Variable & $\mathbf{1}$ & $\mathbf{2}$ & $\mathbf{3}$ \\
\hline High-MPR indicator & $0.16^{*}$ & 0.18 & 0.044 \\
& $(0.078)$ & $(0.11)$ & $(0.12)$ \\
Attend $^{++}$ & $0.065^{* *}$ & $0.082^{* *}$ & 0.10 \\
& $(0.019)$ & $(0.025)$ & $(0.078)$ \\
Age in years $^{+}$ & & 0.083 & -0.27 \\
& & $(0.27)$ & $(0.35)$ \\
Male indicator & & -0.13 & -0.032 \\
& & $(0.11)$ & $(0.12)$ \\
Number of siblings & & 0.12 & 0.35 \\
& & $(0.12)$ & $(0.22)$ \\
Single parent household indicator & & 0.0034 & \\
& & $(0.12)$ & 0.091 \\
Allowance & & & $(0.085)$ \\
TV watching & ++ & & 0.021 \\
& & & $(0.094)$ \\
Church attendance & & 0.082 \\
& & & $(0.091)$ \\
Adjusted $r$-squared & & 111 & 55 \\
\end{tabular}

Notes: Standard errors in parenthesis. An asterisk $\left({ }^{*}\right)$ means significant at the $5 \%$ level; a double asterisk $\left({ }^{* *}\right)$ at $1 \%$; a plus sign $(+)$ indicates log of value; a double plus sign $(++)$ indicates the log of value plus one, to allow for zeros.

cients, with generally somewhat less significance.

The first regression includes an indicator variable for the high MPR sites as well as a measure of the proportion of all the years of schooling a subject has had that were at the school where the experiment was conducted. We use this as a measure of group attachment, or at least of familiarity with the other subjects in the experiment. We set this variable to zero for the summer program students, since students often enroll in these programs somewhere other than their regular schools, and they attend somewhat irregularly. In addition, these programs had been in session less than two weeks when these experiments were conducted. This measure of group attachment has a positive and statistically significant effect on first-round contributions. At the mean of the high MP variable, moving from $25 \%$ to $75 \%$ attendance increases contributions by 0.2 tokens.

In regression 2 we add some variables describing the basic demographic characteristics of the subject and their family. (Because we were not able to collect this data at all the sites, $n$ for this regression and the subsequent one are smaller than in the first regression.) Subject's age, gender, number of siblings, and an indicator variable for singleparent families all are statistically insignificant. We also estimated this model using indicator variables for birth order and only child, allowance, and measures of family income and mother's education. None of these variables had a significant effect. In regression 3 we added descriptive variables that measured allowance, TV watching, and church attendance. ${ }^{6}$ None of these was significant. We also did not find significant effects with measures of participation in organized sports and other organized group activities, such as Boy or Girl Scouts. Regressions that included variables measuring day care attendance at various ages showed that these had no effect on contributions.

The only variables for which we find significant effects on first-round contributions are the marginal private return and our mea-

6. These variables were only available for the observations from the first set of experiments. 
sure of group attachment. These variables both have the expected positive effects. The first effect has been consistently found by others in adults, and the second effect has been documented with adults using experimental treatments, for example in Orbell, van de Kragt, and Dawes [1988.] To our knowledge however, no one has attempted to find an equivalent effect with adults using a measure of attachment developed outside the experimental setting.

On the other hand, a host of other variables describing the subjects and their families have no statistically significant effect on contributions. Since most other public good experiments have been conducted on rather homogeneous groups and have not attempted to collect the sort of descriptive variables that we have, we do not know to what extent this second finding is specific to children. However, slight differences in contributions by gender have been found for adults, e.g., in Andreoni and Vesterlund [1997]. Here, we find no evidence that this effect exists among children. Marwell and Ames [1981] report that training in economics increases free-riding. Here we find no evidence that activities that might plausibly be thought to promote and encourage cooperative behavior, such as church attendance, have any effect on altruistic behavior in this experimental setting.

\section{Behacior across Iterations}

We now look at the pattern of donations across iterations, in order to see whether children learn to free-ride. We begin with descriptive statistics and then present regression results. Figure 1 shows how contributions change with iterations. Looking only at averages, there is little evidence that our subjects learn to free-ride during the experiment. The general pattern is an initial increase in contributions, followed by a leveling off and then a very slight decrease. ${ }^{7}$ Over all sites, average contributions start at 2.1, increase to 3.2 in round 5 , then fall to 3.0 by round 10 .

Figure 2 shows the percentage of subjects giving each amount, for each iteration, with lower amounts toward the bottom of the

7. This pattern was also ohserved in the data from the first set of experiments, so far as they went.

FIGURE 1

Mean Contribution for Different Sites, by Iteration

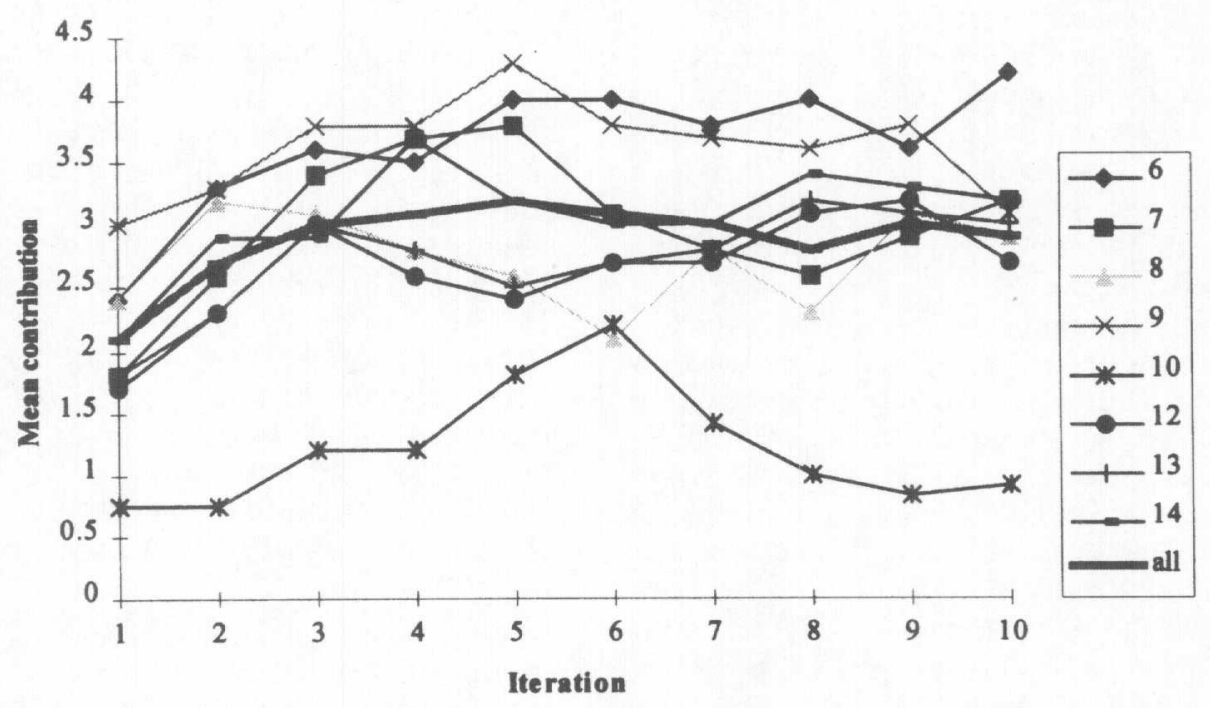


FIGURE 2

Percentage of Subjects Contributing Different Amounts, by Iteration

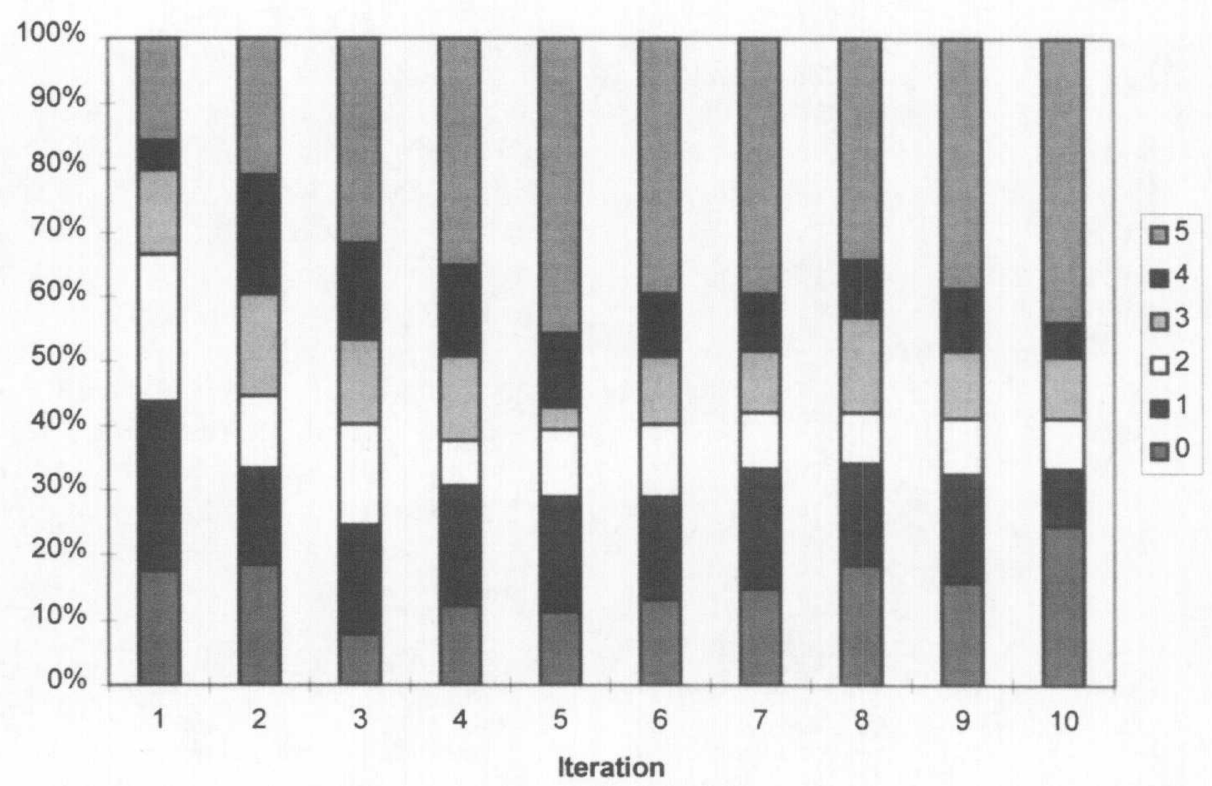

figure. From this figure, it is clear that the initial increase in contributions over the first five rounds comes from both an increase in the number of subjects contributing and an increase in the size of contributions from those already contributing. The proportion of subjects giving nothing falls from $19 \%$ in round 1 to $11 \%$ in round 5 . From round 5 to round 10 , the proportion giving zero rises to $24 \%$. However only three contributors give zero every iteration. Most zero contributions come from small contributors switching to and from zero. The percentage of subjects giving the maximum five tokens increases from $16 \%$ to $44 \%$ over the first five rounds and then stays near that level. One striking difference between first round and tenth round contributions is the number of children contributing more than zero but less than five. In the first round, only about onethird of the subjects either keep all of their tokens or give all of them to the common pool. By the tenth round, approximately two-thirds of the children are either keeping all of their tokens (the Nash equilibrium) or contributing all of them (the Pareto optimum.)
The general rise in contributions is at odds with the large fall in contributions typically, though not always, found with adult subjects. On average, our subjects do not learn to free-ride. However, it is still possible that some subset of subjects does. While $50 \%$ of the subjects gave more in the last three iterations than they gave in the first three, $32 \%$ of them gave less. Still, it would be a mistake to say that these subjects are necessarily free-riding, since random behavior would also produce some subjects with reduced contributions. However, since freeriding is typically found with adults, we hypothesize that it should be more common among our older subjects. We use a panel data model with random effects to test this hypothesis. This model allows for unobserved heterogeneity in the propensity to contribute. ${ }^{8}$ Descriptive statistics for the regression variables are in Table III, and the results are in Table $V$. As in the regressions on first-round contributions, the dependent variable is the $\log$ of the contribution plus

8. Except in regression 3, the Hausman test cannot reject the hypothesis that random effects, rather than fixed cffects is appropriate for this data. 
TABLE V

Contributions Across Repetitions: Random Effects Regression Results

\begin{tabular}{|c|c|c|c|}
\hline \multirow[b]{2}{*}{ Variable } & \multicolumn{3}{|c|}{ Regression } \\
\hline & 1 & 2 & 3 \\
\hline High return site & $\begin{array}{c}0.20^{*} \\
(0.082)\end{array}$ & $\begin{array}{c}0.21^{*} \\
(0.085)\end{array}$ & $\begin{array}{r}0.26^{* *} \\
(0.084)\end{array}$ \\
\hline Iteration $^{+}$ & $\begin{array}{l}0.077^{* *} \\
(0.018)\end{array}$ & $\begin{array}{l}0.66^{* *} \\
(0.20)\end{array}$ & $\begin{array}{l}0.56^{* *} \\
(0.21)\end{array}$ \\
\hline $\begin{array}{l}\text { Attend (percentage of all school } \\
\text { at this school) }\end{array}$ & $\begin{array}{l}0.054 * * \\
(0.019)\end{array}$ & $\begin{array}{l}0.054^{* *} \\
(0.019)\end{array}$ & $\begin{array}{l}0.077^{* *} \\
(0.020)\end{array}$ \\
\hline $\mathrm{Age}^{+}$ & & $\begin{array}{l}0.53^{*} \\
(0.25)\end{array}$ & $\begin{array}{c}0.44 \\
(0.26)\end{array}$ \\
\hline $\mathrm{Age}^{+}$iteration $^{+}$interaction & & $\begin{array}{r}-0.27^{* *} \\
(0.093)\end{array}$ & $\begin{array}{r}-0.23 * * \\
(0.098)\end{array}$ \\
\hline Male & & & $\begin{array}{c}-0.066 \\
(0.085)\end{array}$ \\
\hline Number of siblings ${ }^{++}$ & & & $\begin{array}{c}0.067 \\
(0.099)\end{array}$ \\
\hline Allowance $^{++}$ & & & $\begin{array}{c}0.008 \\
(0.054)\end{array}$ \\
\hline Single parent household & & & $\begin{array}{l}-0.13 \\
(0.099)\end{array}$ \\
\hline Constant & $\begin{array}{l}0.86^{* *} \\
(0.072)\end{array}$ & $\begin{array}{c}-0.29 \\
(0.55)\end{array}$ & $\begin{array}{c}-0.19 \\
(0.58)\end{array}$ \\
\hline$n$ & 126 & 124 & 111 \\
\hline$r$-squared: & & & \\
\hline Within & 0.015 & 0.022 & 0.017 \\
\hline Between & 0.12 & 0.12 & 0.21 \\
\hline Overall & 0.073 & 0.077 & 0.12 \\
\hline$P$-value for Hausman test of RE & 1.0 & 1.0 & 1.0 \\
\hline
\end{tabular}

Notes: Standard errors in parenthesis. An asterisk $\left({ }^{*}\right)$ means significant at the $5 \%$ level; a double asterisk means significant at the $1 \%$ level; a plus sign $(+)$ indicates log of value; a double plus sign indicates the log of value plus one, to handle zeros.

one, and independent variables are transformed as noted.

Regression 1 considers the effects of the MPR, the iteration number, and the attendance measure. All are positive and significant. Regression 2 adds age, and an ageround interaction term. Age is significant and positive, while the interaction effect is significant and negative, indicating that, although older children are initially more generous than younger ones, they also learn to free-ride more quickly. Regression 3 adds some subject characteristics as in the firstround regressions. Again, neither gender, the number of siblings, allowance nor single parent household has a statistically significant effect on contributions. The age, round, and interaction coefficients change only by small amounts across regressions 1,2 , and 3 . As in the first-round-only regressions, we also esti- mated these models using untransformed data, getting the same signs on the statistically significant coefficients, and generally somewhat less significance. We also ran a model with both linear and quadratic terms for round, with similar results.

In general, these results corroborate those in the first-round-only analysis. With the exception of group attachment and now age, and the age round interaction term, none of the variables describing subjects and their families had any explanatory power. We do find that the variables altered during the experiments have explanatory power. The high-return variable remains positive, and in most of the regressions contributions increase over rounds. The positive sign on iteration suggests that, overall, children do not learn to free-ride. In fact, the opposite seems to be true, and they tend to give more 
over time. However the negative coefficient on the age iteration interaction variable indicates that the contributions from older children do decline with repetition. In short, like adults, older children do learn to free-ride.

The estimates from regression 2 show that iteration has a negative effect on donations for children 11.5 years old or older. For a hypothetical 8 year old with characteristics that are at the means of the other variables, our estimates predict that donations would increase by 0.2 tokens from the first to the second round, and by 0.12 from the second to the third round. For a 14 year old, they predict decreases of 0.14 and 0.08 , respectively. An heroic extrapolation predicts that 21 year olds would decrease their contributions by 0.5 tokens from the first to the second round and by 0.28 from the second to the third.

It is tempting to conclude from the results of this section that young children are not only basically altruistic, but in fact that they are better than adults at maintaining mutually beneficial behavior. This may well be true, and one plausible reason for it might be social ties. The coefficient on the variable measuring attendance is positive in both the regressions on first-round contributions and the regressions on all contributions. If children in general have stronger social ties than do adults, they may be better at maintaining mutually beneficial cooperation. However, an alternative explanation for our results is that the repeated linear public goods game may be a poor method of measuring altruism.

In the linear public goods game, mistakes cannot be distinguished from altruism. Because children are presumably more likely than adults to make mistakes, this experiment should therefore tend to overestimate children's altruism relative to that of adults. One reason for repeating the game is to give the subjects a chance to learn. with the idea that once they understand the game their actions will accurately represent their preferences. However, it is apparent from the literature that it takes many iterations for adults to learn free-riding, and it seems likely that children have even more difficulty. The result that donations decline with repetition for older children, but not younger ones, may simply mean that older children learn more quickly with repetition than do younger ones.

\section{REVEALED PREFERENCE EXPERIMENT}

As a check on the possibility that some of our subjects are fundamentally confused, and that the behavior we are observing is unrelated to altruism, at three sites we followed the public good experiment with a second test of altruistic behavior. (Subjects were not informed that this would be done until after the conclusion of the public good experiment.) In this section, we discuss this revealed preference test. We then give withinsubject comparisons of the results of the revealed preference experiments with those from the public good experiments.

The experiment, based on Andreoni and Miller [1998], tests whether subjects" choices about how much to give to another person obey the axioms of revealed preference. To the extent they do, we argue that their giving behavior in this experiment is rational and therefore that it can be used to provide a check on behavior in the linear public goods experiment.

The experiment is essentially a modified dictator game where each subject is given some tokens and an opportunity to pass some, all, or none to an anonymous partner. The modification is that each subject is shown 11 possible budget constraints for this transfer, each with a different income and price ratio. The budget constraints were selected to have many integer bundles, and to cross in many places. The constraints we used are shown in Figure 3, and the bundles presented on the sheets are shown with dots. We explain that only one randomly chosen transfer will actually be made and also that they will be on the receiving end of transfers, if any, from a different partner than the one to whom they can give tokens.

Since many of our subjects cannot do the math necessary to stay within a budget, we presented the possible transfers visually. We prepared a separate sheet of paper for each budget constraint, showing each of the possible integer combinations of tokens they could keep for themselves and tokens they could pass to their partner along that budget constraint. Every child received the same 11 pages, ordered randomly. Subjects were given 11 star stickers, and were told to put one star on each page, placing the star in the box that showed the combination of tokens for themselves and tokens for their partner that they 
FIGURE 3

Budget Sets with Available Bundles Indicated as Dots

\section{Tokens Given}

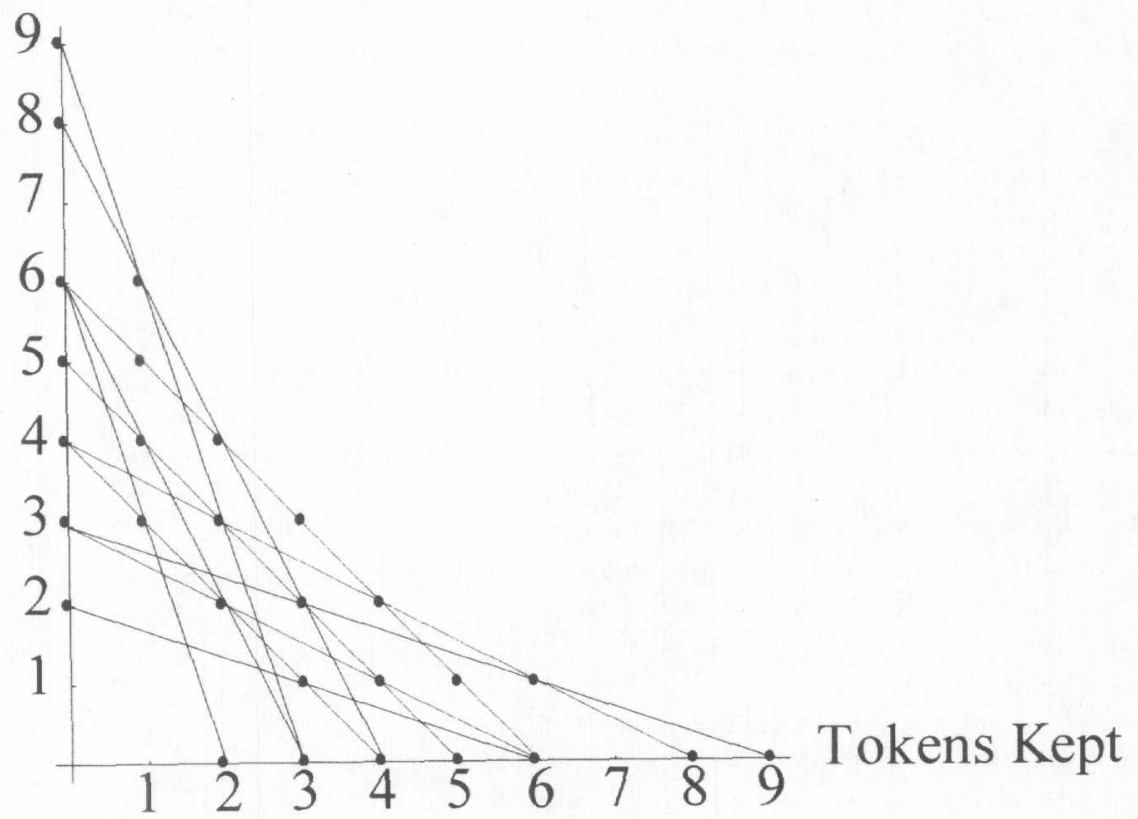

liked best. The subjects seemed to understand this procedure very well.

\section{Revealed Preference Results}

Using an algorithm based on Varian [1995], we counted the number of violations of the generalized axiom of revealed preference (GARP) found in each child's eleven choices. A violation of GARP occurs when a subject picks a bundle of goods $x$ when they could have picked another bundle $x^{\prime}$ that they have indirectly revealed they preferred to $x$. Bundle $x^{\prime}$ is directly revealed preferred to $x$ if $x^{\prime}$ is chosen when $x$, or some other bundle with more of at least one good than $x$, is in the choice set, and $x^{\prime}$ is indirectly revealed to be preferred to $x$ when some chain of directly revealed choices leads from $x^{\prime}$ to $x$. In addition to just counting the violations, we calculated their severity using Afriat's [1972] efficiency index, or AEI. This index ranges from zero to one. The amount by which the index falls below one can be thought of as the proportion of income that the subject wasted by failing to choose rationally. For example, a AEI of 0.75 means that the cheapest available bundle that would have satisfied the axioms of revealed preference would have cost only $75 \%$ as much as the bundle they actually chose, which did not satisfy the axioms. We used the incomes and prices implicit in the budget sets to construct this measure."

In addition to checking for GARP violations, we compute a simple measure of the willingness to share in this experiment. The measure, which we call "proportion shared," is simply the average over the 11 constraints of the number of tokens in the chosen bundle that go to the partner, divided by the total number of tokens in the bundle. Sum-

9. Because these choice sets are discreet, rather than continuous, this measure potentially exaggerates the severity of violations, since the subject might have actually chosen a bundle with a higher AEI. had it been available in the choice set. 
TABLE VI

Revealed Preference Summary Statistics

\begin{tabular}{llclc}
\hline Variable & Mean & Standard Deviation & Min & Max \\
\hline All subjects (40 observations): & & & & \\
$\quad$ Violations & 2.2 & 3.0 & 0 & 11 \\
AEI & 0.87 & 0.20 & 0.33 & 1 \\
$\quad$ Proportion shared & 0.29 & 0.25 & 0.0 & 0.98 \\
"Rational" subjects (28 observations): & & & & \\
$\quad$ Violations & 0.43 & 0.82 & 0 & 2 \\
AEI & 0.97 & 0.049 & 0.875 & 1 \\
Proportion of tokens shared & 0.22 & 0.24 & 0 & 0.98 \\
\hline
\end{tabular}

mary statistics for this measure are given in Table VI along with those for the number of violation and the AEI.

The average number of violations is 2.2 , and the average severity of the violations is 0.87 . We ran computer simulations picking bundles randomly and found an average of 5.7 violations and a severity index of 0.65 . Our sample was skewed: 22 subjects had no violations. Clearly our subjects are not choosing randomly. We regressed age and gender on the number of violations and the AEI, and neither variable was significant.

We arbitrarily classify the 28 subjects with AEI scores of 0.875 or above and no more than two violations as "rational." 10 These subjects averaged 0.43 revealed preference violations and had an average AEI of 0.97 .

10. Running the regressions with all 40 subjects gave similar results.
On average, they picked bundles where they shared $22 \%$ of the available tokens, compared to an average for all subjects of $29 \%$. We tested whether the demographic and other characteristics that we had tried in the previous regressions to explain public good contributions could explain altruism in this protocol. None of these variables had statistically significant effects on the proportion shared in this experiment. (The school attendance variable did not vary over these subjects, all of whom were at summer programs.)

Table VII shows the results we obtained when we regressed these dictator game results on contributions in the public good experiment, using two-sided tobit to account for the fact that contributions must be between 0 and 5 . We only use the 28 "rational" subjects in these regressions. We run this regression with two different dependent variables: the amount shared in the first round

\section{TABLE VII}

Regressions Using Revealed Preference Results

\begin{tabular}{lcc}
\hline Independent Variable & $\begin{array}{c}\text { Regression 1 } \\
\text { Dependent Variable } \\
\text { 1st-Round } \\
\text { Contribution }\end{array}$ & $\begin{array}{c}\text { Regression 2 } \\
\text { Dependent Variable } \\
\text { 10th-Round } \\
\text { Contribution }\end{array}$ \\
\hline Tokens shared divided by & $6.8^{* *}$ & 11.3 \\
all tokens in bundle & $(2.4)$ & $(5.9)$ \\
$n$ & 28 & 28 \\
Pseudo $r$-squared & 0.086 & 0.027 \\
\hline
\end{tabular}

Notes: Standard errors in parenthesis. An asterisk $\left({ }^{*}\right)$ means significant at the $5 \%$ level; a double asterisk $\left({ }^{* *}\right)$ means significant at $1 \%$. 
of the public good experiment and the amount shared in the last round. The results show that behavior in the dictator game is related to behavior in the first round of the public good experiment, but not in the last round, although neither $r$-squared is particularly high. Note that the dictator game immediately followed the last round of the public good experiment.

We propose the following interpretation of these results, which we believe is also consistent with the results of the public good experiment. Behavior in the first round of the public good experiment is to some extent driven by altruism, though there also seems to be a significant amount of confusion. While with adults the confusion generally is reduced with repetition, and the less altruistic subjects start to free-ride once they understand the true incentives, many children simply get more confused with repetition. By the last round, the behavior of a significant fraction of the subjects is driven by confusion. In the subsequent, simpler, revealed preference experiment, that confusion is eliminated. Subjects who gave large amounts in the last round of the public good experiment were not generous in the subsequent revealed preference game. However, we should note that another interpretation is that the children are acting optimally in the public goods game by building cooperative behavior and that in the subsequent dictator game, since there are no opportunities for further cooperation, they then reduce their contributions.

\section{v. CONCLUSION}

We find that first-round contributions by children, both in terms of the mean and the variation, are in line with those that others have found with adults. This is encouraging, especially since age has only a small effect. The marginal private return and a measure of group attachment have positive effects on these contributions. Other subject and parent demographic variables explain none of the variation in these contributions.

The overall increase with repetition is the opposite of that generally found in other experiments: one of the stronger effects that those studying adults have found is that contributions fall with repetition. Since the Nash equilibrium for these experiments is zero contributions, this general downward trend is a comforting tendency, suggesting that the subjects are at least converging toward a Nash equilibrium. In our experiments, we observe increasing contributions, the wrong direction from the point of view of most learning models. One plausible explanation for this behavior is that younger children mistakenly view their contributions as investments with high rates of return, whereas older children learn to free-ride, as adults do.

However, despite their difficulty learning to free-ride, we argue that even young children clearly do have a taste for altruism. Even in the dictator game, they give an average of $29 \%$ of their tokens away. Furthermore, their choices about how many tokens to give away generally, though not always, change rationally in response to changes in their incomes and the price of giving. This is true both across subjects in the public good experiment and within subjects in the dictator game. Similarly, children with longer attendance at the same school give more. Still, children's preferences about altruism do seem substantially less well formed than do adults. Although their results are not directly comparable (we use more budget constraints, each with fewer choices) Andreoni and Miller [1998] find that only 13 of 142 adult subjects had any GARP violations, while 18 of our 40 subjects did.

We believe that the finding that primary school children act in a way that is not drastically different than that of adults is robust and somewhat surprising. Retrospection alone is enough to convince most people that other sorts of behaviors change drastically between childhood and adulthoodchoice over time, for example. However, on further thought, it seems appropriate that the altruistic behavior of children would be similar to that of adults: both live in very social environments with repeated interactions with others and with many opportunities for others to observe their behavior and reward or punish them for it. Our subjects are already old enough to have received a large amount of encouragement to engage in sharing activities, and have experienced the advantages, and disadvantages, of altruism in many different settings. 


\section{APPENDIX EXPERIMENTAL PROTOCOLS}

\section{LINEAR PUBLIC GOOD PROTOCOL}

We first had the subjects gather around a table and read the following instructions to them.

We are going to play a game. By playing the game, you will earn tokens that you cian use to buy things at our store. (Show store.) Each white token that you get during the game is worth about 10 cents, and in addition you may get some red tokens that are worth about 3 cents. Pay very careful attention to these instructions, because the better you understand them the more tokens you can carn, and the more tokens you have the more things you will be able to buy. If you have questions raise your hand. Otherwise, please be quiet and listen carefully, just like you would to your teacher in school.

You will get 10 turns at the game. At the beginning of each turn we will give you 5 white tokens, and you may get more tokens at the end of cach turn. depending on your decisions and the decisions of the people in your group. Remember, the more tokens you have at the end, the more stuff you can buy, so pay careful attention to these instructions! You are going to be in a group with 5 other kids. You will be in the same group during the whole experiment. We are not going to tell you who is in which group, and we aren't going to tell anyone else who is in your group. We are going to keep this a secret even after the experiment is over, so no one will ever know.

You get to decide whether you are going to keep your tokens or are going to share them with your group. We do not think it would be better for you to share the tokens or better to keep them. No one will know how many you shared or how many you kept or whether you shared or not. Keep your decision a secret: you are not allowed to ask other kids what they did, or tell them what you did. If you do, we may come and take a token away from you!

You will be sitting at these desks. Each desk will have a "keeping cup" and a brown envelope in front of it. The keeping cup is for your tokens, and the envelope is for the ones that you want to share. At the beginning of each round, we will give you 5 ncw tokens. You will put the ones that you want to share in your envelope, and the ones that you want to keep in your cup. You can share any number of tokens, from zero to 5 . The partitions are there so that you can keep your choice a secret. When everybody has made their choice we will collect all the envelopes.

Now, I will tell you how the sharing works. I will add 3 more tokens for every 1 that kids share. So, what if I get these six envelopes back? (Experimenter then dumps out six envelopes that have one token each) See, each envelope had one token in it. Now there are six tokens in this group's pile. (Experimenter then counts out the ones that are added.) Now there are 24 in the pile. So now I need to split this up to the six kids: each one gets four tokens back. I will put 4 tokens in each envelope, and deliver them back. These kids would then put the tokens in their keeping cups.
Let's try that again. So, what if I get these six envelopes back? (Experimenter dumps out six envelopes that have $0,1,1,0,3,0$ tokens in them.) $\mathrm{OK}$. now there are five tokens in this group's pile, so I add three more five times. Now there's 20 in the pile. Each kid gets 3 back, and I have 2 left over. See these red tokens? Three reds equal one white. So I am going to make some change, just like 5 pennics make one nickel. (Count out, 3 reds for each white) Now I have six reds. so each kid can get one. I will put 3 whites plus 1 red in each envelope.

Remember: If you get a red one hack, it means just part of a white one. ALL the kids in each group get back exactly the same number of tokens. no matter how many they share or keep. Notice that the kids who shared nothing got just as many tokens back as the kid who shared 3.

We then had the subjects sit down at tables, with tri-fold partitions for pritacy, and read them following.

There are five white tokens at your place. These tokens belong to you. You may keep all of them if you want, or you can share some or all of them with your group. We do not think it would be better to share or to keep, we are just interested in what you decide to do.

Decide how many tokens you want to keep and put them in your cup. Put the number of tokens you want to share in your envelope. When you are ready raise your hand so at helper can pick up your envelope. Don 1 let anyone else see how many you are sharing or keeping!

Remember: For every token that you and the other kids in your group share, I will add 3 more tokens. Then I will divide all the tokens up equally. If you get a red one back, it's as if I tried to divide up a white token in three parts.

Rencmber: You might get back more than you shared. but you might get back less. It all depends on what the other kids in your group do.

We then collected the entelopes, recorded the contributions, and passed back the eamings. The following was read affer each subsequent round.

OK. now let's play another turn. A helper will give you back your envelope with the tokens you get from your group's pile, and another helper will give vou five new tokens. You can't share any of the tokens that you get back in your envelope, so count them, quietly, and then put them in your cup right away. You can share as many of your new tokens as you want to. Raise your hand when you're ready to have your envelope picked up. The same five other kids are still in your group. They don't know whether you are in their group, or how many you shared last time.

\section{After the ninth round, we also read the following.}

The game is almost over. You have only one more turn to play this game.

\section{After the tenth round, we read this.}

We hope you enjoyed the game. Now we want you to start counting out your tokens. We will hold up the things from the store one at a time. If you want to buy something, hold up your hand and we will bring it around to you. Thanks for helping us. 


\section{GARP PROTOCOL}

The GARP experiment was performed before subjects were allowed to buy things from their public good experiment eamings. Subjects remained seated at the stations used in the public goods experiment, and we read the following instractions to them.

This is a game that gives you a chance to share with another person. We are interested in finding out what you want to do---there is no "right" thing to do, just do whatever makes you feel hest.

The game is pretty complicated, so pay careful attention. The tokens that you get can be used to buy things from our store, just like in the last game. Remember that the more tokens you get the more you can buy at the store.

The game works like this. We will match you with 2 other kids here today. We are not going to tell you who they are, and we aren't going to tell them who you are. It will be impossible for any kid to cver know this. Onc of these kids is your "giving partner" and the other kid is your "getting partner."

We are giving each onc of you a packet with 11 pages. (Pass these out.) Turn over the first sheet, and look at the next page. Fach page has some boxes on it. Each box has some circles and some squares in it. The circles stand for tokens that will go to you, and the squares stand for tokens that will go to your giving partner. On each page, you will pick just one of these boxes. Remember: circle tokens, if any, will go to you, and square lokens, if any, will go to your "giving partner". Your "getting partner" will also be picking a box, and so vou will also get the square tokens, if any, from the box that your "getting partner" chooses. See-you have a partner and you are also someone else's partner.

Remember, each token is worth about 10 cents. So, if you choose a box like () it's just like kecping ( ) for yoursclf. and giving ( ) to your "giving partner." And if your getting partner chose this box. then he would get ( ) and you would get ( ).

You pick a box by taking one of your stickers and putting it in whichever box you want to. But remember, you only have one sticker for each page, so you can only choose one box on each page. You have to put just one sticker on each piece of paper. If you don't do this, you might not get any tokens.

However, while we want you to make a choice on every page. the game is a little more complicated. After cverybody has picked one box from each piece of paper. we will put a ticket for each page in my hat and I will close my eves and pick out one ticket. That is going to decide which page we are going to give tokens for. You will get the round tokens. if any. from the box you picked on that page, and the square tokens. if any. from the box that your "getting partner" picked on that page. The square tokens. if any, from the box that you picked will go to your "giving partner." Since nobody knows which ticket will come out of the hat the best thing to do is pick the box you like hest on every single page.

So there are two ways to get tokens: you get the round ones, if any, from the box that you choose, and you will get the square ones, if any, from the box that your partner decides to give to you.
Remember: I might pick the ticket for any page from my hat. So pick the box carefully on every single pieco of paper.

We then drew a ticket from the hat, paid the subjects and partners accordingly, and allowed them to spend the lokens as described abole.

\section{REFERENCES}

Afriat, Sidney. "Efficiency Estimation of Production Functions." Intemational Economic Reriew. October $1972,568-98$.

Andreoni, James. "Cooperation in Public-Goods Experiments: Kindness or Confusion?" American Economic Reriew. September 1995, 891-904.

Andreoni, James, and John Miller. "Giving According to GARP: An Experimental Study of Rationality and Altruism." Social Systems Research Instituto Working Paper 9902. University of WisconsinMadison, 1998

Andreoni, James, and Lise Vesterlund. "Which Is the Fair Sex" Gender Differenees in Altruism." Social Systems Research Institute Working Paper 9810, University of Wisconsin-Madison, 1998.

Eisenberg, Nancy, and Paul H. Mussen. The Roots of Prosocial Behalior in Children. Cambridge: Cambridge University Press. 1989.

Grusec, Joan E: "The Socialization of Altruism," in The Derelopment of Prosocial Bcharior, edited by Nancy Eiscnberg. New York: Academic Press, 1982 $139-66$.

Ledyard, John O. "Public Goods." in The Handbook of Experimental Economics. edited by John H. Kagel and Alvin E. Roth. Princeton. N.J.: Princeton University Press. 1995, 111-94.

Marwell, Gerald, and Ruth E. Ames. "Economists Free Ride. Does Anyone Else?" Joumal of Public Economics, June $1981,295-310$

Orbell, J., A. van de Kragt, and R. Dawes. "Explaining Discussion Induced Cooperation." Joumal of Personality and Social Pstcholog, May 1988, 811-19.

Palfrey. Thomas, and Jeffrey Prisbrey. "Anomalous Behavior in Lincar Public Goods Experiments: How Much and Why?" American Economic Reriew. December 1997, 829-46.

Peters, H. Elizabeth, A. Sinan Unur. Jeremy Clark, and William Schulze. "Frec-Riding and the Provision of Public Goods in the Family: An Experimental Test of the Rotten Kid Theorem". Cornell Department of Economics Working Paper. 1997.

Underwood, Bill, and Bert S. Moore. "The Generality of Altruism in Children," in The Detelopment of Prosocial Behatior, cdited by Nancy Eisenberg. New York: Academic Press. 1982, 25-52.

Varian, Hal. "Efficiency in Production and Consumption," Working Paper and Mathematica Notebook 1995 [online]. Available: ftp://ftp.telospub.com/ pub/EconFinance/CompEcon/ [January 12, 1999]. 